Sewenmergel und =Ralf, burd bie verjutedenen Shiditen bes Grinjanb: fteins unb bes Sarattentalfes ift biefe Itrbeit bis zu ben bunflen Anter freibeibidten vorgeiffitten. Dabei ift ber $3 \mathrm{ug}$ ber flamm lein geraber,

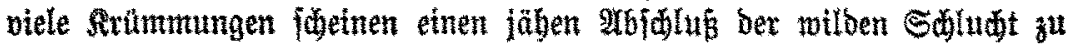

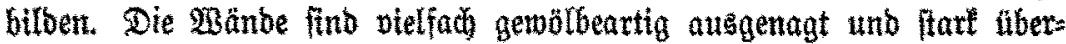
Gängenb. Daj itbrigens bie tojenben fluten now mandiestual ben in vermeintlid fiderer bobe angelegten Pfar erretien, bas zeigte ein in

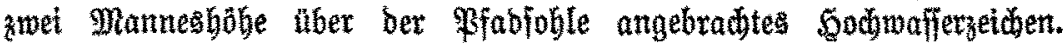

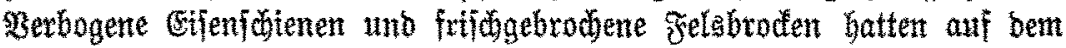

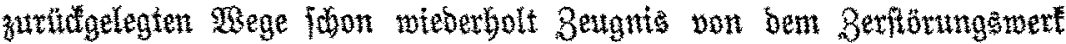
ber Brettad in ben Gunitagen bes vorigen Sabres gegefen. Den $926=$ fălub ber Rlanmmanberung bilbet ber \$Baffertall. - Droben in ber Sobe weben bie weibblaue uno bie idwargelbe Fabne: wit treten auf bas Gebtet bes Doppelablers über und erretden nach turzer Wanberung bie Baljeridanze, ein ehemals ofterreidifiches Bolthaus. Son bet Terraffe

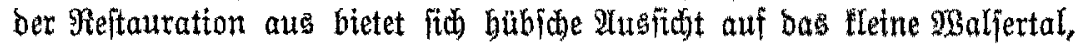
rüdtwäts ber bohen Sfen und bie Gottesadernärtbe, betbe mit ben be= fannten Sarenfeldern bes Sctrattenfalfes.

Rad) bem Mittageffen murbe bie $\mathfrak{B a n b e r u n g}$ auf jebr gut gebaltenen

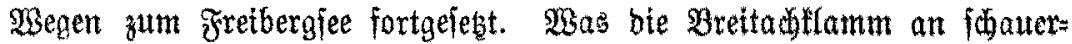

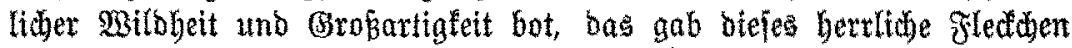
Erbe an übermältigender 2 xnmut unb Söbubeit. Den bintergrunb bilbet

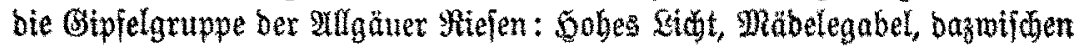

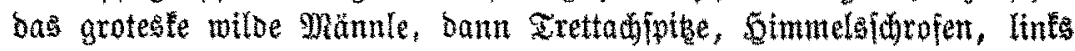
fortjegent zur wilben 50 fat uno zutu Mebelhorn.

Dann ging's binab gen Dberftborf, Ginuber uber bie wilbe Stillad unb vorbet an ber Borettofapelle, vorbei an bem lururibfat Sotel Wittelebacherhof unb bem einfaden Sagbgaus unjeres \$rinzregentent.

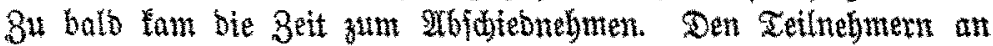

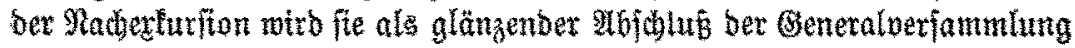
1911 in Iteber Erinnerung bleiben.

Rotling.

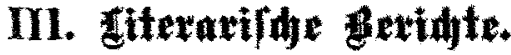

Nr. 78.

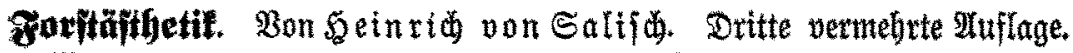
Mit 133 Textabbilbungen. Berlin, Berlag von Sultus Epringer, 1911. 434 S.

Bet zmet Sauptwerfammlungen bes beutionen Foritwereines ftand bie 
"Fortäfihetit" auf ber Tagesoronung: 1905 in Darmitabt, wo von

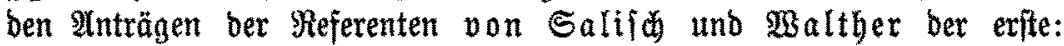
"Die Bewirtidaftung ber Balbungen nach Sabonbettsrudfituten ift als ein in ben mirtidaftlichen unb jozialen Berbältniffen ber Neuzeit be= grünbetes Debürfints anzuleben" einftimmig angenommen wurbe, unb 1906 in Danzig, wo ber vom Foritmirtfafatsrate vorgeiblagene Sab

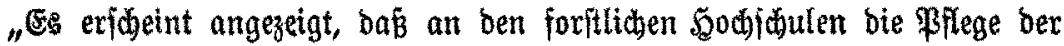
Walbesföbönbeit in afabemijaten Borträgen behandelt wirb" faft all= gemeine 3uftimmung fanb. Wienn nun auth ber weitere IIntrag, bie zu= ftänbigen Minifterien ber Cinzelftaaten zu exfuchen, bie aybbaltung be-

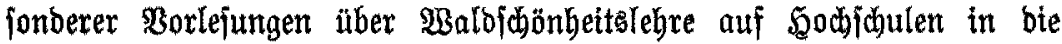
\$ege zu leiten, nidht ben gleiden crofolg batte, fo mupten es bie

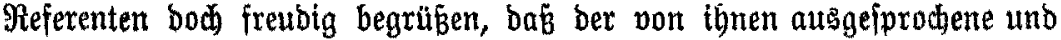
näber begrimbete Gebanfe unter ben Foritleuten uno Balbbefisern einer früber faum geafinten Simpatbie begegnete.

Unterbefien if bon ber 1885 zum exftenmal exidienenen Forit = äfthetil von jenes allgemeine, nidt ohne Berbienfit Des Berfafiers geiteigerte Snterefle an ber Sache faum notwendig geworben wäte. Benn aud bie Ein= teilung bes Stoffes bie gleiche geblieben ift, wie fie bei Bejpred)ung ber zweiten Muflage (Fortitw. Centralblatt, 1902, 5.525 mit 527) mitgeteilt wurbe, fo fanb bod wieber eine erbeblide räumlidje und ladlidide $\mathbb{E} r=$ weiterung bes Budes unt eine weitere Bercidjerung mit Tert= abbilbungen ftatt.

Der Berfaffer beföräntt jeine beachtenswerten $\mathfrak{A}$ tusfübrungen nidht

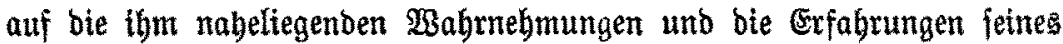
eigenen Befthes uno jeiner engeren Seimat; begreiflichermeije nebmen aber

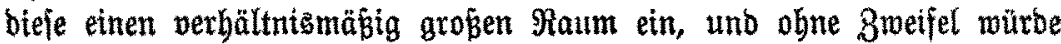

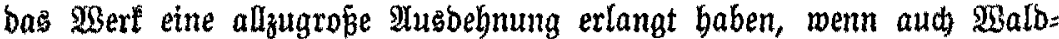
bilber uno Birtiogafteformen aus andern Gebieten ebenlo beullid ge= iđgitlbert worben wären.

Mebr um ben auberorbentfichen Reidfum bes Begenftantes zu beleudten, als um Sritil an bem Gebotenen zat üben, möd) ein fübbeutioner Forfmann einiges Benige anführen, was ex im $I$. Teile, ben $(\mathcal{G r u n b}$ : lagen ber Forftäfthetif, unter B. Swonbeit ber Matur vermif́t hat.

Im 8. Sapitel über ben "äfthetificen wert ber $\mathfrak{g}$ olgarten" bätten wohl auch jene herborrageno fob̆nten Cruppen unb Einzelitämme ebler Raubhölzer, beionbers bes Bergahorn, Erwäbnumg verbient, bie im bayerifagen 5odgebirge, tetíneife aud im "Bayerifhen $\mathfrak{B a l b e}$ auf 
Wiejenfluren, an Balbrämbern, auf Mlplidutungen und Beibejidachen fo angenebm ing sluge fallen.

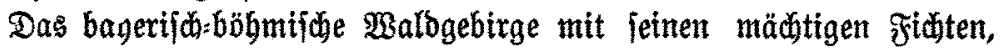
Tannen uno Buthen follte als bejonders reidibaltiges Sebiet fojoner Walbbäume in $\Re a p .11$ unter ben "Raturbenlmälern aus ber beutid)en Baumwelt" genannt jein.

Nebenbei jei auf Den fleinen, vielleidgt von ber bemughten Duelle veridulbeten Šrrtum aufmerkfam gemacht, ber barin liegt, baßs bie als "ältefter Deutjōer Baum" Gildich bargeitellte und im Terte S. 108 u. 143 erwäbnte Eibe im "Berggünble" im 2thgäu mit ber Stanbortshäbe $1520 \mathrm{~m}$ über bem Meere angegeben ift, wäbrento fie auf einer 50 be von $1125 \mathrm{~m}$ (ס. \%. 3853 bayer. Fuß $)$ fteht, wie aus ben jebr zuverläffigen "Begetationèverbältnififen Sübbayern" (ङ. 519) von Dtto Sendtner zu erieben.

Störender berührt aber eine Qüffe im II. Teile "2l ngewendetc Forfäfthetif". Sm 16. Sap. fino bie, Betriebsarten in ibrer

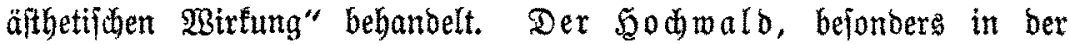
Form des überbaltwaldes wirb allen andern Betriebsarten, aud bem \$itentermalbe vorgezogen. Nidgt genannt mirb aber jene Betriebsart, bie

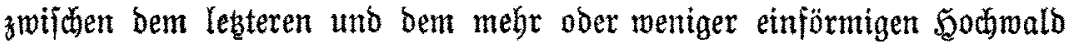
mit Rabliblägen liegt unb bie gerabe bie jöñiten Maldbilder bietet der geregelte zemelidglag und insbejonbere ber horftweife fomel= ¡dlag, wie er in Bayern uno auth anderwärts in Sildbeutifland üblid ijt.

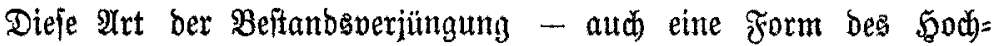
walbes - bat fid bort natürlich entwicelelt, ift von tübohtigen Foritleuten gefördert und ausgebilbet und von bervorragenden Männern ber $\mathfrak{W i}$ ifen= ¡Gaft, bejonders pon Rarl Gayer inftematifid begrünbet und gelehrt worden; fie hat ith jeit lange bewährt und auber ibren walbbaulidoen Borzägen Walbbilder hervorgebraut, bie jeben Frorftäfthetifer befriedigen

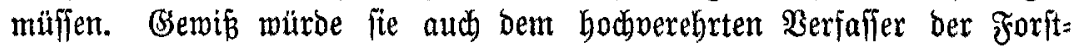
äfthetif zulagen, ber fid im 19. אap. Felbft als einen Freund ber natürlið̌en Berjüngung befennt, wo nur bie Berbältniffe ihr Ge: lingen fther erboffen laffen. Şn unjern aus Fidften, Tamnen und Bublen gemifdten Baldungen gibt es für ben Erfolg bes Goritweifen ober jaumiblagmeifen Zemelbetriebes nur eine Gefahr, bie Motwendigleit allzu rajuer Mlbnułung ber in Berjüngung begriffenen Beitände.

5gerr von Salifh weift in einzelnen Fällen wieberbolt nad), baß

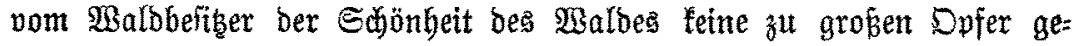

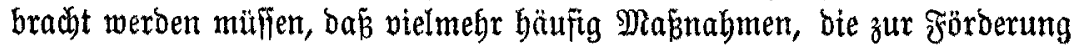


Der Malbesfdbönheit beitragen, aud Borteile für ben Mubenießer bieten. unbefannt mag ihm wohl bei Serauggabe bes Budes ber Borwurf ge= wejen fein, ber neueroings ber bejifichen Staatsforitverwaltung gemacht

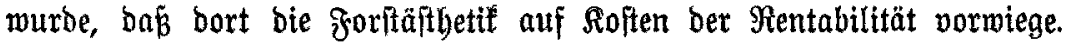
(Foritto. Centralblatt, 1911, Sheft 5, Biterarifde Beridate: "Die Brob̉. Seffitiche Staateforitwirtidaif ${ }^{\prime \prime}$ ).

Der Berfafier der Foritäftbetil verlangt aub̆ fonjt vom $\mathfrak{B a l d b}$ befizer in exfter Rinie Rüdfidt auf Den Ertrag. Ex fteht jogar bem Boben= reinertrag nidgt feinbfelig gegenüber. Er verlangt mit Wilbrano u. a.

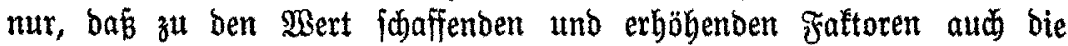
Sdjönheit unb bie Erhaltung ber Sabnhbeit bes $\mathfrak{B a l b e s}$ mit in bie

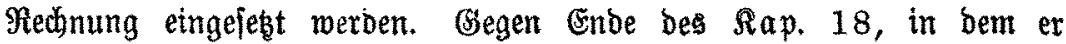
biejen Gegenftand bebandelt, finben fï̈ bie Morte: „,, 0 man nun ein

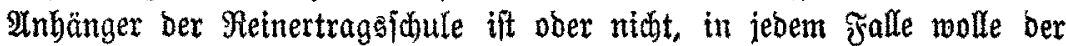
Foritmann feinen idealen Sinn feithalten." Bezeidyneno in biefem Sinne ift auch bie im $\Re$ ap. 29 bebanbelte unb näher begründete $\mathfrak{d n t e r i d j e i d u n g ~}$

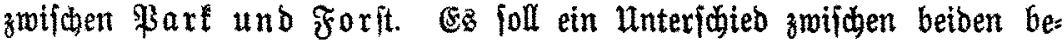
fteben unb aud äuperfic erfennbar fein; ben Parf wie bie parfartige Gartenanlage grianbet uno bebanbelt ber Lanbidaftsgärtner, wenn auch ber Foritmann gerne bazu beigezogen uno wenn ihm aud häufig bie Briinbung ,freier $\mathfrak{A n l a g e n " ~ u n t ~ v o n ~ M l l e e n ~ u ̈ b e r t r a g e n ~ w i r b . ~}$

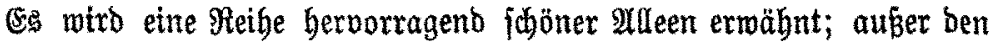

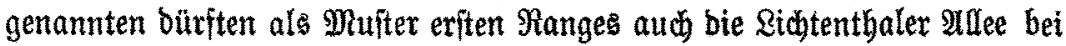
Baden= Baben und bie von Salzburg nach Sellbrunn Erwähnung verbienen.

Sn einem Salupporte fajt von Salij巾 noch einmal hurz zu= jammen, was ex unter bem, "Bejen der Forft funft" verjtebt: "Die Sauptiache ift funitgerechter $\mathfrak{B i r t i c h a f t s b e t r i e b , ~ b i e ~}$ Pflege ber ftanborts= gemäßen Baum= uılo Straudbarten, bte Segung altehrwüroiger Bäume, bie Erfalließ̧ung ber Beftänbe burd Bege, bie in bas Snnere hinein=

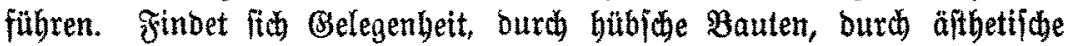
Geftaltung von Biefen unb Gewäfiern, burch Zelsblöede und Steine Schmud binzuzufügen, fo foll man fie nid)t veriäumen.

Tlusblicfe mögen Dem $\mathfrak{A}$ uge geftatten, Daß̧ es in bie Ferne jưweife."

Wie boch der Beriafjer bie $\mathfrak{A}$ ufgabe einjdät, bie bem Forftmanne wie bem Balbbefither zugebacht ift, geht aus bem Schlubiake bes leksten, bes 39. Rap. bervor, wo er jagt: "Der Foritmann joll nidgt nur bie Saböngeit pflegen, er foll aud bie Walbbefuther baza erzieben, bie bar= gebotene Malbespradbt zu verfteben uno zu wärbigen."

Dazu gebört aber Sinn unb Reritänonis für bas Sajone; unt foldes zu gewinnen unb zu erböben, eignet fich faum etwas anberes 
nuebr als bas Studium bes vorliegenden Buthes in feiner neuen $\mathfrak{A} u \mathfrak{f}=$ lage, bie wir baber bem foritliden $\mathfrak{B u b l i f u m}$ wie aud ben $\mathfrak{B a l b b e f t}$ ern aufs märmite empfeblen fönnen.

raesfeldt.

กx. 79.

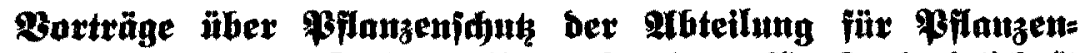

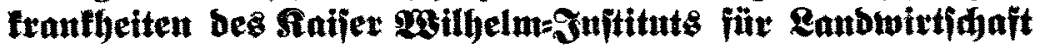
in Bromberg. Šerautgegeben von Dr. Şander. 3meites Sheft: Forftidu. Mit 52 Iextabbilloungen. Berlin, Berlag von $\mathfrak{B a u l}$ Parey, 1911, 94 S. Freis 1,60 M. 25 Stü $35 \mathscr{M} .50$ Stüu 60. Ah.

Mie bei Befpredfung bes exften מ̃eftes mitgeteilt, ${ }^{1}$ werben von oben genauntem Snftitut Rurie über Pflanzenichus für \&anomirte, Forft= wirte, Walsbelizer abgebalten unb neuerbings bie bort gehaltenen $\mathfrak{B} o r=$

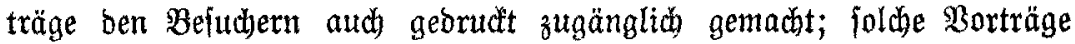
bietet benn auch bas vorliegende 2. พ̌eft. Daşflbe entbält:

1. Cinen $\mathfrak{B o r t r a g}$ von $\Re e g=$ und Foritrat $\mathfrak{S}$ errmann in Danzig über, Die Bebeutung Der Samengeminnung für bie Ergiehung gejunder Pflanzen in ber Forftwirtidaft", in welchem er bieje zurzeit fo aftuelle Frage eingeheno uno unter Anführung alles beffen, was hierïber jeitens zahlreicher Frorfaber bis jeşt feîtgeitellt murbe, bejpricht. Errflärlichermeife ift es intbejondere bie $\mathfrak{B r o v e n t e n}$ bes Riefernfamena, bie einen breiten

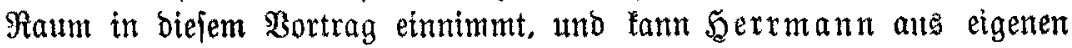

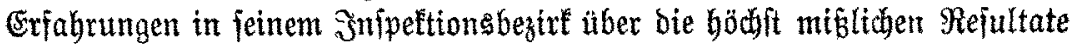
beridhten, welde bie Bermendung von Riefernjamen jübfranzöitjoer und weitungarifider Gerkinft ergeben haben.

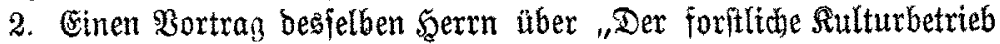
unb feine nidbt parafitärent und burd̆ pflanzliche Schäblinge erzeugten Sranfleiten mit bejonberer Berüdfiobtigung ber Riefer". Der Bortragende erörtert bie Frage bes sutturbetriebs int allgemeinen: Samenbefdaffung, Pflanzenerziehung, Befäbrbung ber Sulturen burd) atmofphärif be Einflüfe,

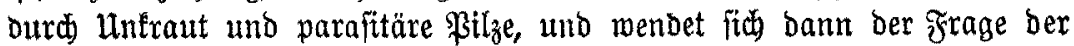

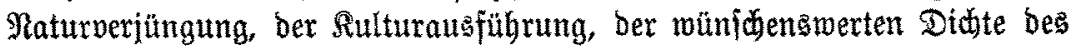
Pflanzenitandes zu, mobet aus naheliegenden Grünben bie Siejer im Mittelpuntt bee Jntereffes fteht.

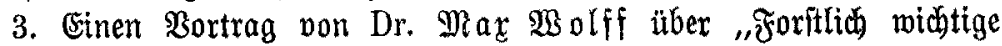
Sđjilbläufe", bem zablreidhe Abbildungen beigegeben finto. Wern aud

3) Forftw. Centralbl. 1910, ङ. 613 . 
ber Froritmann nidbt leidft mit Deren $\mathfrak{B} \in$ fämpfung zul tun haben wirb, fo ift boch eine Renntnis biejer in zablreichen Formen unt oft auffallender Ericheinung im $\mathfrak{B a l b}$ vortommenden Snjettengruppe für ihn von Snterefie.

Der billige ßrets ber gut ausgeitatteten Sdyrift oürfte beren wünljøenswerter Berbreitung jörberlich jein.

Nix. 80.

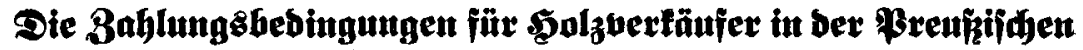

Etantsporfitwerwaltung. 3 ujammengeitellt, erläutert und fritijob betradtet von \&. Sdilling, \$rofeffor ber Foritwirtidaft an ber Foritafademie Eberswalde \$erlag bes , 54 S. Broich. Preis $5 \mathscr{M}$.

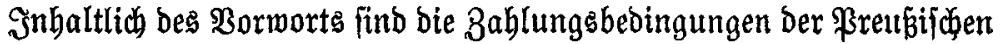
Staatsioritberwaltung im Raufe ber Beit burch ftete Rachträge und Er:

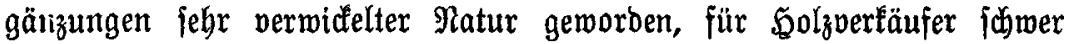
veritändlich und jelbit fïr ben Bermaltungsbeamten fowwierig zu hand:

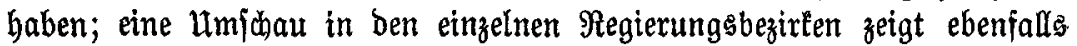

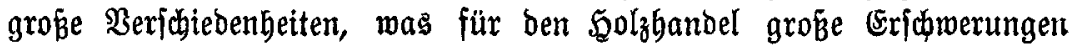
bringen $\mathfrak{m u} \tilde{\beta}$.

Die vorliegende Sdrift ift aus bem $\mathfrak{B u n} j$ d entitanden, an ber $\mathfrak{B} e=$

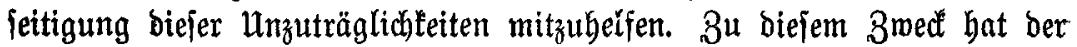
Berfafier fich bejtrebt, jene Beftimmungen möglicbit foitematifo zujammen= zuftellen, ihnen bie nötigen Erläuterungen anzufügen und burch Ber:

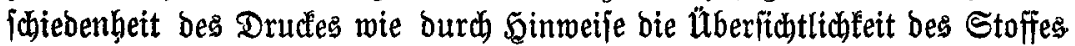
zu förbern und basfelbe bierburch auch für bie Praxts braudbaar zu macken.

Sin einer Sdylubbetradtung gibt Swilling jodann eine Zujammen= jaffung beffen, was er für münjhenswert und nötig hält: eine Robi=

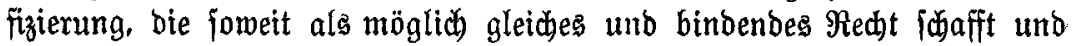
bie vielfachen $\mathfrak{u n f l a r b e i t e n , ~ b i e ~ u n z m e i f e l b a f t ~ b e i t t e h e n , ~ b e j e i t i g t . ~} \mathfrak{S}^{\mathrm{u}}$ folcher Robifizierung bürfte er burch jeine Sdyrift eine wertwolle $\mathfrak{B}$ orarbeit geliefert haben.

Dr. Fürit.

Mr. 81.

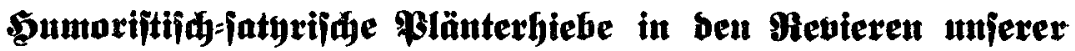

Joxitzeit. 3ur Beluftigung für Deutichlands edle Baldbrüber auf=

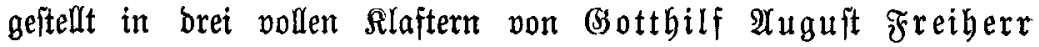
vou Maltib, orb. Mitglied ber herzogl. Sachjen=(Sothaifhen unb

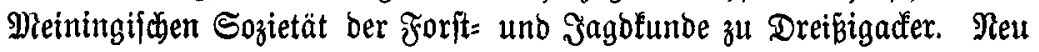




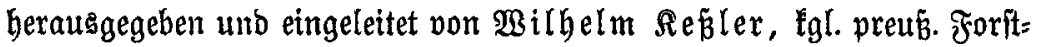
meifter a. D. Reubamm, Berfag von $\Im$. Neumann, 1911. $317 \mathrm{~S}$. Preis brofd. $3 \mathscr{M}$, in $\$$ appband 3,50 $\mathscr{M}$, geb. $5 \mathscr{M}$.

In einer längeren Einleitung gibt uns ber Şerausgeber einen Rebens: abrí bes Freiherrn von Maltiz, ber nach in Tharand vollenbeten Stubien fich 1822 ber Sberförfterprüfung in $\mathfrak{B r e u k e n ~ u n t e r z o g , ~ j e o b c h ~}$ infolge zmeier gegen höhere Frortbeamte gerichtete Spottidbriften alabald aus bem Staatsbienft entlaffen wurbe und fich pobann bis zu jeinem 1837 erfolgten früben $\mathfrak{I} 0 \mathrm{~d}$ als Sdyriftiteller auf perichiedenen Bsebieten der Riteratur betätigte. Rach Reß̉lex's Urteil war er zwar nur ein

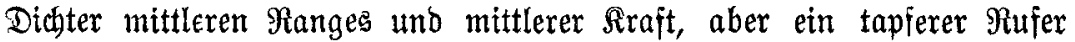
im Streit ber Getfter, etn wacterer ebrlider Rämpfer für feine über= zeugung, voll marmer Riebe für fein Bolf uno Baterland.

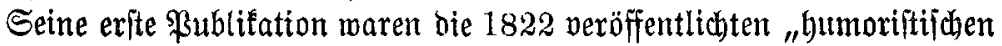

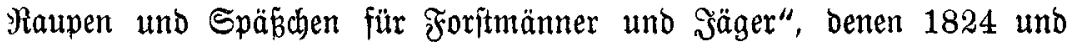
1827 zwei weitere "Rlafter" folgten; fie iheinen 2 Intlang gefunden zu haben, Denn 1828 gab er fie gejammelt und nermehrt als 3. Aluflage unter Dem oben genannten Titel heraus.

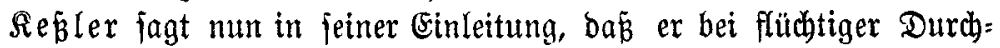

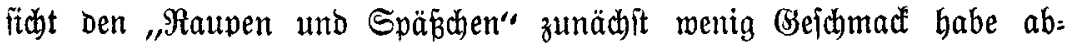
geminnen fönnen, baßj er aber bei näherer Befanntichaft mit bem Didbter und feinen anderweiten Sibriften bod anderer $2 \mathfrak{A}$ fitht geworben fei, in oem etwas fraulen Ranfengewirr feiner forftlichen Raupen boch noch mancbe buftende Blüte, manche gejunde Frucht gefunben und Darum ber Unregung bes Reumann'iben Berlages zur Ilusgrabung eines $\mathfrak{B e r}=$ ichollenen Folge geleiftet habe.

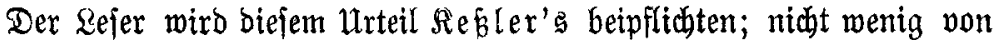
bem, was uns als Şumor ober Satyre vorgejeţt wirb, entipricht unjerem jeţigen Gsejdymad nur teilweife - aber anderes vermag nodh jekgt unjer

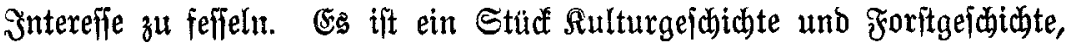

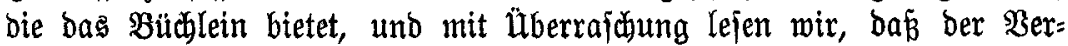

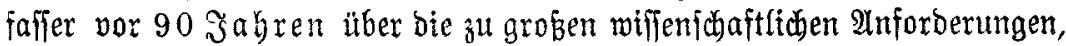
Das übertriebene Sdyreibwejen in Der Foritwerwaltung klagt, wäbrend boch wir älteren Männer ber Gegenmart glauben, dáp es bamit zat

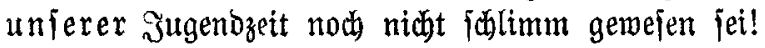

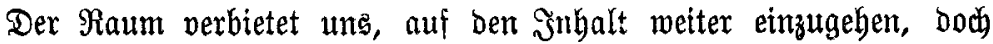
glauben mix das sBüdlein allen Fadbgenoffen empfeblen ză fönnen, die einmal ein paar Stunden ftatt erniter Biffenidgaft einer amüjanten Seftüre mibmen mollen.

Dr. Für rit. 


\section{Mr. 82.}

Mitteilungen aus bet Stant8forituerwaltung Bayerns. Sूeraugs, gegeben vom $\mathfrak{R g l}$. Staatsmmifterium ber Finanzen, Minifterialforft: abteilung. 12. Soeft. Münden 1910.

Dem vorliegenden 12. Sefte, einem ltattlidjen Band von 215 Seiten, entrebmen wir nachtebent eine ßeibe von Bablen, bie allgemeineres Snterefie Gaben bürften, und fügen einzelnen bie in bem erïten biejer feit 1901 eriçeinenden ftatiftichen Seite für frubhere Jabre unb $\mathfrak{J a b r}=$ zebnte (1868-1898) mitgeteilten 3ahlen vergleidssmeile an.

Die Staatewaldfläche Bayerns beträgt 940393 ha, barunter 114304 ha improbuttives Terrain (vormiegend im \$odhgebirg); fie ift feit 1868 um runo 15000 ha burd Infauf gewadjen.

Bur $\mathfrak{B}$ erwertung gelangten im \$ahre 1908

$1941637 \mathrm{fm} \mathfrak{N u b h o l}=54,7 \%$ Der angefallenen $\mathfrak{g}$ olzmaffe,

1607644 "Brentyolz $=45,3$ " "

mit einem Bejamterlös von $49080383 \mathscr{M}$, wobei ber Entgang butra \$olzabgaben auf Berechtigung 1751488 beziffert.

Es beträgt biernach ber ફolzanfall pro beltar $4,31 \mathrm{fm}$, ber Erlös 51,24 (bezw. intl. jenes Entgange 53,91) Marf.

Diejen 8ablen ftebt im Sabr 1898 gegenüber ein 2 nfall von

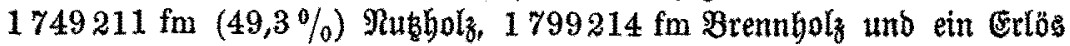
von $34759152 \mathscr{M}$ - bie auberorbentliaje Steigerung bes Erlöję̧ fällt in bie Plugen.

Die Eimnabme als Rebennukungen beträgt $1168259 \mathscr{H}$, ber

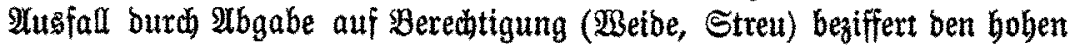
Betrag von $939145 \mathscr{M}$.

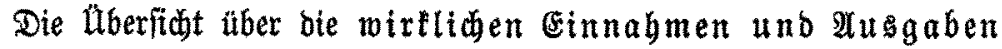
für 1908 weift naw

eine Einnabme von 51447651 M

"2utggabe "23713240,

eine Reineinnahme von $27734411 \mathscr{M}$ ober $33,72 \mathscr{A}$ pro Sgeftar.

Sier bürfte mun ein Bergleich mit früberen Эabrzebnten von Snterefie jein. Şt ben Эabren 1877 bis 1885 betrugen bie Reineinnabmen 10-12 Millionen Marf, um bann allmăblicy auf 16 Millionen im Jahre 1894, auf 19,692 Millionen im Jabre 1898 anzumadjen uno bis zum Sabre 1908 auf ben oben angegebenen bohen \$etrag zu fteigen.

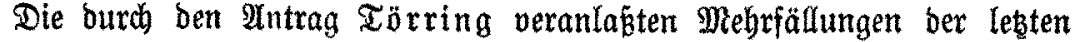
Sahre werben eine abermalige nidjt unwejentlicfe Erhöhung ber Ein= nahmen bringen - bie hohe Beoeutung ber Staatewaldungent für ben Staat birrfte hierdurch genügend beleudtet jein! 
Die 2luggaben für Forftelulturen find von 1406686 M im

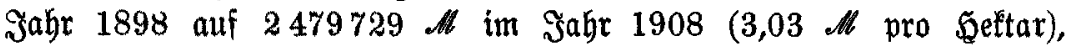
jene für $\mathfrak{B}$ Begbauten von $1454357 \mathscr{M}$ auf $2196497 \mathscr{M}(2,35 \mathscr{M}$ pro Seftar) geftiegen - Zahlen, weldje beweifen, bå jene Bedeutung auch feitens bes \&anbtages burd (Semährung reidjerer Mittel anerfannt wirb.

2(uch auf bem (Gebiete ber Forftrechtseinlöiung, bie in Bayern befanntlid nux im Bege gütlichen übereinfommens erfolgen fann, iit laut

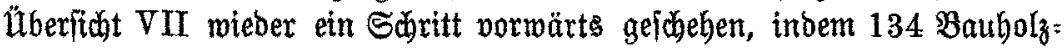
refte, 2504 Brennholzredte, 35 Strent= unb Weiberechte mit einem Betrag von 1589169 abgelöf murben. Wie bedeutend bie Belaftung ber bayerifden Staatswaloungen trob ftetiger Ablojungen nod ift, zeigt eine überfitht aus bem Jahy 1895, bie beren Rapitalwert auf 75 Millionen Mart angibt.

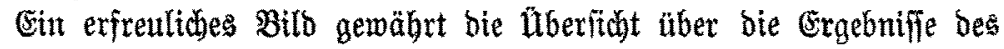
Forffitrafwefens, indem fie bie ftetige abnabme ber Foritfrevel und Foritpolizetïbertretungen nadmeift. Die $\mathcal{B}^{a b l}$ ber $\mathfrak{A n z e i g e n ~ b e t r u g ~ i m ~}$ Jahr 1868 aus Stantsmaloungen 93583 , aus niaht ärartalifiben Walbungen 85118 , bis zum $\mathfrak{3 a h r} 1898$ war beren 3 abl bereits auf 28291 bez̧w. 25208 ftetig zatrüdgegangen uno 10 Sahire fpäter auf 25610 bezw. 20588 gejunten - auf runb ben vierten Teil jener eriten 3 ahlen!

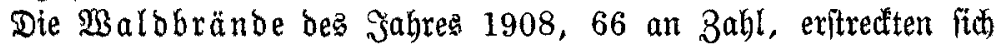
utber 177 ha, fo bas auf 5294 ha der Staatemalofläthe 1 ha Brano. flädthe entfällt. Bon biejen Balbbränden maren 53 reines Bobenfeuer, 10 Bobenfeuer mit Gipfel= und 3 mit Stammfeuer.

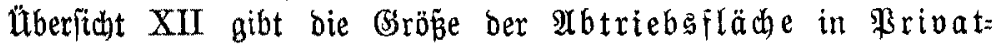
waldungen in ben Jabren 1897 bis 1908 auf 74804 ha, jeme ber Bieberaufforftungen auf 77802 ha an; auperbem wurben nod

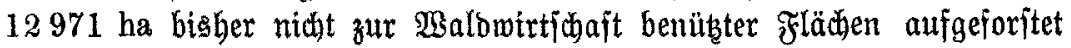

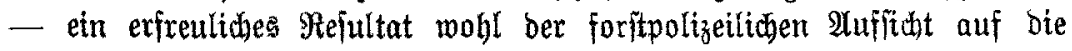
\$rivatwaldungen uno bex ftaatliden $\mathfrak{x}$ ätiglett zur 5 ebung ber Frivatwald: wirtichaft.

Die überfidyt über bie unentgeltlide Rranlenfürforge für bie Staatoforfarbeiter im Sabre 1909 weift den bohen Betrag von

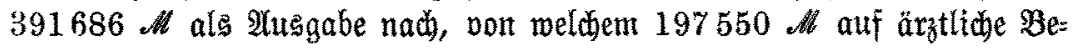
banblung, 41098 Ah auf $\mathfrak{A r z}$ neifoften, $128000 \mathscr{M}$ auf Srantengelder,

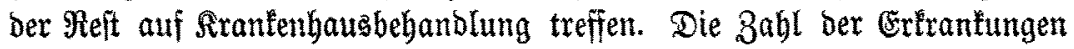
mit je 54 auf 100 Bollarbeiter uno 8122 in ganzen erideint als eine fehr bebeutende.

Uuberbem bringt bas beft 12 nod) eine Bufammenitellung ber 
Fâllungèergebniffe, Foritfulturen uno Wegbauten in ben Gemeinbe: uno Stiftungswalbungen, bann ber Durdifinittaerlöie aus ber \$olzoerwertung in Den Staatsmalbungen nach Gorittämtern und Sortimenten, fowie ber bort erwadjienen Bewinmungafoiten.

Mr. 83.

Vocabulaire Forestier Français-Allemand-Anglais, von $\$$. (S) eridel, Durdigejehen von 2 . $\Re$. Fif - Drforo, Clarendon Press - 1911.

Das vorliegende Sanbmörterbuch in Tajöenbudjormat, bas nunmebr bie fünīte $\mathfrak{A}$ uflage erreidgt hat, gibt in 3 Mbteilungen

$$
\begin{aligned}
& \text { Franzäjifj - Englifich - Deutich - } 51 \text { Seiten } \\
& \text { Deutfa - Franzojitid - Englijw - } 78 \text { " } \\
& \text { Engliich - Franzöfitio - Deutich - } 63 \text { " }
\end{aligned}
$$

einen umfangreidjen Teil bes forftlichen $\mathfrak{3 0 r t i d h a k e s , ~ e b e n i o ~ b e s ~ b e r ~}$ F̧orit= und Sagbzoologie. (Segenüber ben früberen Auflagen baben $\mathfrak{Z} e=$

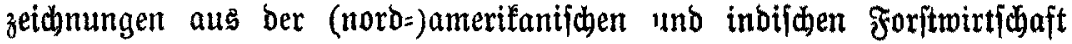

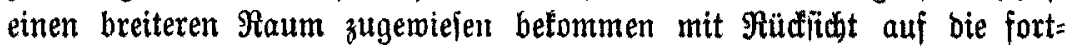

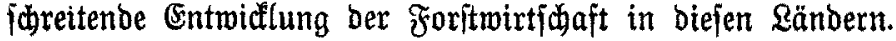

Beim Stubium ber in Bitradjt fommenoen Fadfliteratur wie auf

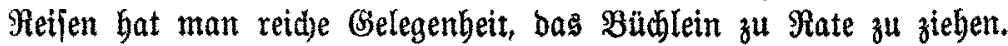

Mr. 84.

Principles of Handling Woodlands, von Henry Solon Graves, Chief Forester, Forest Service, U. S. Dep. of Agriculture. I. Auflage, I. Tausend - New York, John Wiley \& Sons, 1911.

Staat, Staaten und Private ber Bereinigten Staaten von 9 Imerifa find in Den leketen Sabren bemüht, in ihren forftlidien Maß̧nahmen ben (Srunbjägen ber Forftwirtidaft, wie fie in ber alten $\mathfrak{B e l t}$ fabn längft befteben, zu folgen ober ibnen minbeftens näberzutreten. Trobbem fehlt es an zujammenfaffenden Werfen über zoritmirtichaft, ba bas bisher über amerifanijobe Froritmirtichaft Eridienene nur jragmentarifo in ver=

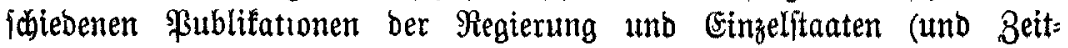

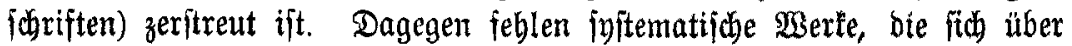
bie veridiedenen Gebiete der Froritwirtidaft verbreiten und für bie Sand Des Eigentümers von $\mathfrak{B a l d u n g e n ~ b e i t i m m t ~ f i n d . ~ D i e j e m ~} \mathfrak{a} b e l$ wollen

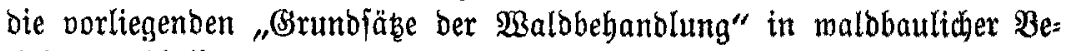
ziebung abhelfen. 
Das $\mathfrak{B e r t}$ foll nidt eine volffänbige Mbhanblung über Walobau in allen feinen 8 weigen fein, fonbern betrifft zunächit bie Behanblung

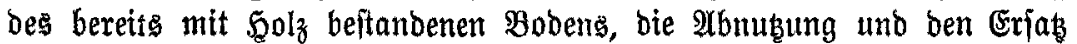
ber reifen Beftänbe, Berbefjerungshtebe in nod) nicht für bie $\mathfrak{A}$ trt reifen Beitänden unb ben Sounc biejer Raldungen, inghejonoere gegen Feuer. Nidut behandelt find f. B. bas Säen und $\mathfrak{F}$ flanzen, welches einem andern Autor zur Bebandlung vorbehalten iit.

Da bie in Betradht fommenden malbbauliden Methoben vielfach mur primitio fein tönnen, fino aud foldhe in exfter ginie berituffichtigt worben.

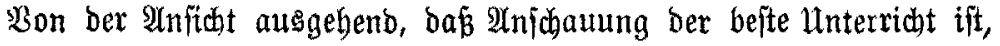
ift bas Wert febr reid) mit Radbilloungen von \$hotographien aus= geftattet, welche bie oberfte Forftbehörbe ber Bereinigten Staaten bem Berfaffer zur $\mathfrak{B}$ erfügung ftellte.

Die Alusitattung bes Berfes ift jehr gut. $\quad$-rto-

Mr. 85.

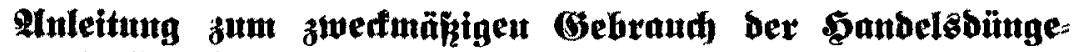

mittel. Bon Theodor Bonsmann. Fö̈nfte 2 Utflage. 44. bis 49. Taujenb. Mit 42 Mbbilbungen im Text. Preis gebunden $3 \mathscr{M}$.

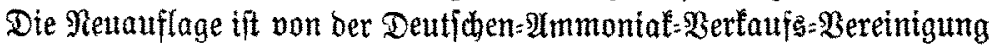
(G. m. b. 5. in Bochum beforgt worben. Die bewäbrte Dispoition murbe beibehalten, ber Text ergänzt uno mit vielen neuen, leiber nidht immer

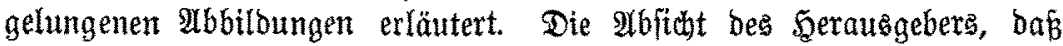
bas Buth „ben Rantwirt über bas Mährftoffbedürfnis feiner Pflanzen aufflären, ihn zur felbftändigen Beurteilung der einzelnen Düngemittel unb zu ihrer żved̈mäbigen Berwendung befähigen folle", bätte vielleidht bie rezeptmäbigen Dingungsanweijungen weriger notwenbig gemadit.

Man wiro itch aud nidft wundern, bá bas jönefelfaure Itmmoniaf als $N=$ Düngemittel an bevorzugter Stelle fteht und fein Şauptfonfurrent, ber Sbilijalpeter, im allgemeinen idjledbt abjobneibet - bas findet wohl umgefebrt ebenjo ftatt. So barf man aud nidht fo ernit nehmen, wenn man S. $132 \mathrm{im}$ Rapitel über "Dïngung und Foriffulturen" lieft "von Sticfifoffoungern fommt bagegen Ehilifalpeter nicht in Betradbt". - Simmerbin veritintmen jolche Einjettigleiten ben fritijchen Rejer und bas ift jichabe,

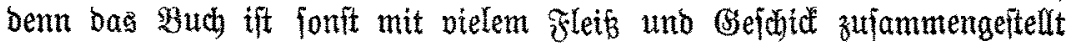

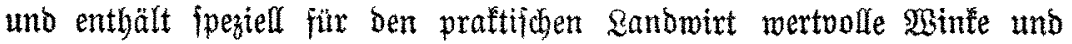
mannigfache gute Ratichläge.

Sarlsuhbe i. B., 1. September $1911 . \quad$ Frof. Dr. Sgelbig. 
शr. 86.

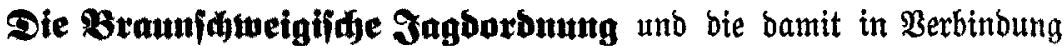

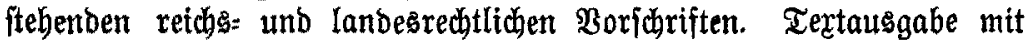
furzen Erläuterungen und Sadperzeichnis, von Dr. F. BS rundner,

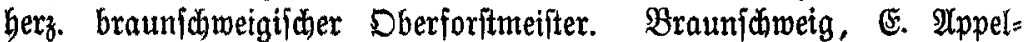
hans \& Comp., 1911. 246 S.

Das im Werzogtum Braunjowneig geltenbe Jagbrent erbielt feine Brundage burch bas Gelet vom 8. September 1848 und beffen $\mathfrak{E r}=$

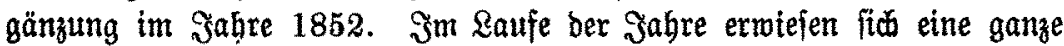
Rethe von Abänberungen unb Ergänzungen als notwendig, auch bas

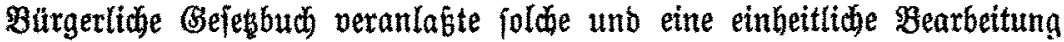
bes Ranbeyjagorechts eridbien baher ichon längit als wünjbenewert. Die Rage bes rings von Preupen eingeidloffenen Sandes madte babei eine

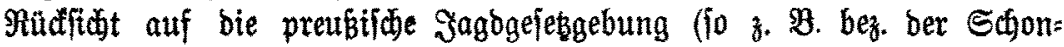

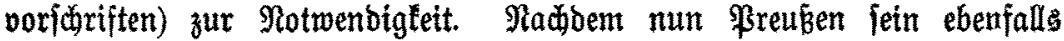
in einer größseren $\mathfrak{A n z a b l}$ von Belesen zerftreutes Jagbrecht burd bie Sagoorbnung vont 15. Suli 1907 zufammengefabt hatte, trat aud bie

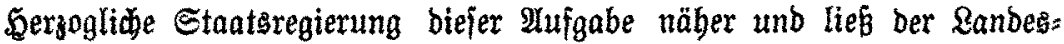
verfammlung ben Entwurf einer Sagborbnung nebit Begrünoung zu= gehen, ber nach eingehender Präfung und Beratung am 3. März 1911 zum Bejeb erboben wurde.

Arls Referent und Fegierungsfommiffar fungierte bierbei Oberforft: meifter Dr. Granoner, aus beffen Freber das vorliegende Büblein ftammt. Dasfelbe umfábt neben ber Jagooronung und ben jebem

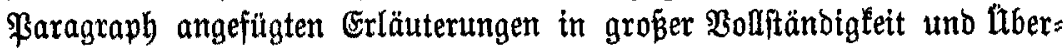

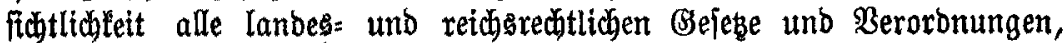

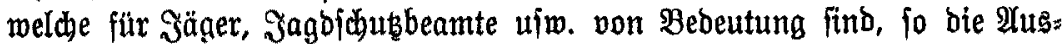

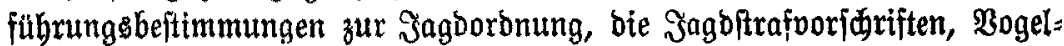
föugejekgebung, Boridriften für bie Jagbidubbeamten, fabrläffige

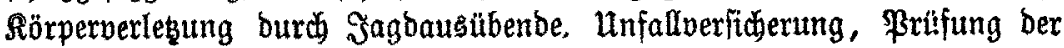
Sanbfeuerwaffen u. ogl. $\mathfrak{m}$. Ein ausfübrlides Sachnerzeidnis exmöglidit eine rajbe Drientierung, und fo wirb bas jorgfältig bearbeitete Bert allen, bie irgenowie mit ber $\mathfrak{y} a g$ uno jagbreditlichen Fragen fu tun baben, eine willfommene Erideinung fein.

Dr. Für it.

$\mathfrak{N r} .87$.

Dr. Mqurih SBillomm's Bilberatlas bes Pfanzetreidis tach bem Engler'iden Evftem. Reu herauggegeben von \$rofeffor E. Söbne. 5. volfftänbig umgearbeitete Iluflage. 526 Pflanzenbilber auf 124 Farbendructtafeln, 1 Sdjwarzbrữtafel und 205 Setten Tett mit 100 
Abbiloungen. Rexifonformat. Bolftänoig in 25 Rieferungen à $0,50 \mu$, geb. $14 \mathscr{M}$. Efftingen uno Münden, Berlag von $\$$. F. Schreiber.

Bon biejem fiton früber angezeigten Rieferungsmert liegen uns nun bie weiteren \&ieferungen $7-15$ vor, mit benen nun $2 / 3$ bes 2 Berfes eridienen fint. Die neuen sieferungen fabliesen iid ben vorber= gegangenen würdig an uno tragen ben im Titel ausgejprodenen 3 wedt, in eriter Sinie ein Bilderatlas zu jein, burch gute Ausführung ber $\mathfrak{A b}=$ bilbungen, beren fith ftets je $3-5$ auf einer Iafel finden, in vollem Make Redhung.

Der $\mathfrak{F r e t s}$ des Berfes mu仿 gegenüber bem, was es bietet, als ein fehr mäb̂́iger bezeidhnet werden.

r.

\section{Nr. 88}

Termitenlehen auf Ceylon. Neute Stubien zur Soziologie ber Tiere, zugletd) ein Rapttel Folonialer Forftentomologie. Bon $\mathfrak{R}$. Ef derich, Dr. med. et phil., o. Profeffior ber 3oologie an ber Forftafademie Tharanot. Mit einem foftematifden 2 (nbang mit Beiträgen von A. Forel, Mils Solmgren, $\mathfrak{B}$. Midaelien, F. Shimmer, F. Silvefter unb E. Wa smann. Mit 3 Tafeln unb 68 Abbiloungen im Text. Jena, Berlag von Guftav Fijuer, 1911. XXXII u. 262 ธ. Preis broid. 6,50 $\mathscr{M}$, geb. 7,50 $\mathscr{M}$.

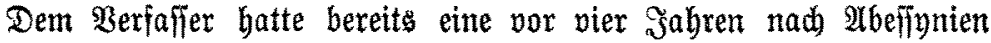
auggejübrte Reife Gelegenhett geboten, "die $\mathfrak{B u n b e r w e l t ~ b e r ~ T e r m i t e n = ~}$ fitaaten zu idauten und zu beftaunen", uno gab ibm bies Beranlafiung, tich eingebenber mit bem Stubium biejer Tiere zu bejäätigen. Eine zweite, im WBinter bezm. Frübjabr 1910 auģgefübrte Reije mit 8 wödent=

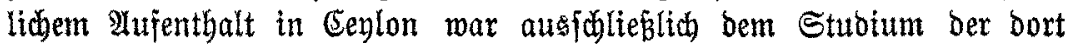
fehr verbreiteten Termiten gemibmet und lieferte inm reiche 9 Hebeute -

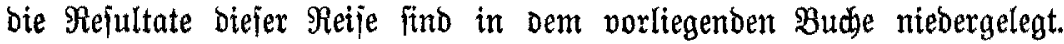

Dr. E. fpridyt auf Grund ber auf Eenlon gemachten Erfabrungen

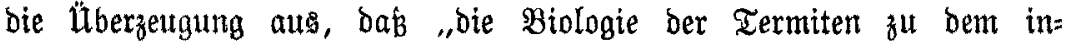
terefianteften Sapitel tierifcher Rebensfunde überhaupt gehört unb zmeifellos Den Sulminationspuntt Des fozialen Tierlebens baritellt" - die Termiten übertreffen Gierin nach jeiner 2Inficht jogar bie ihnen in vielen Stüden

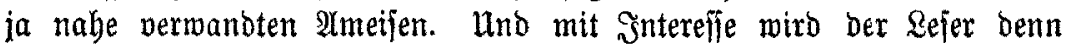
auch bem Berfaffer folgen, ber nacb einer kurzen Schilberung jeiner ßieife

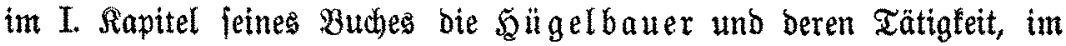
II. Rapitel bie $\mathfrak{R}$ artonfabrifanten, welche nicht gleich ben eriteren bie befannten Fü̈gel erridten, bejpridut. Das III. Rapitel bringt veriditebene Beobadtungen und Berjude im Raboratorium, fo ingbejonbere bez. 
ber mertwürbigen Röniginnen unb beren Rebenstweife, fowie ber Rämpfe

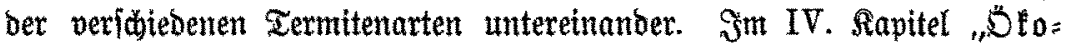

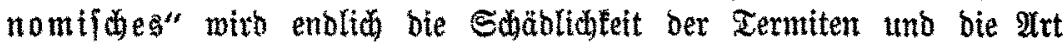

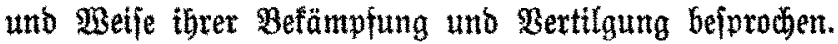

Gin fyitematifder $\mathfrak{A}$ nbang mit Mitteilungen ber im Titel genannten

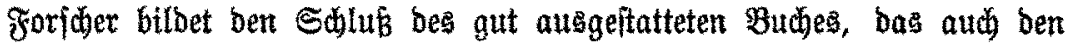
Nidibt:Boologen feffeln wiro.

$\boldsymbol{r}$.

शr. 89.

Gabbe's Grianenzunt. 4. Yuft. neu bearbeitet unb erweitert von A. Staffel II, fgl. Fajanenjäger in Entenfang bei Milbpart. Mit 21 Tertabbilbungen. Berlin, Berlag von $\mathfrak{B a u l}$ Barey, 1911. 155 ङ. \$reis geb. 2,50 M.

Der Berf. hat einer Iufforberung ber Berlagabudibanblung folgenb eine Reubearbeitung bes zulebt im Sahre 1895 erjotenenten Buthes vor= genommen und fich bemüht, bie wäbrenb 30 jähriger Sätigteit tn töniglicten Fafanerien gefammelten Erfahrungen babei entiprechend żu

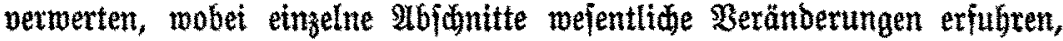

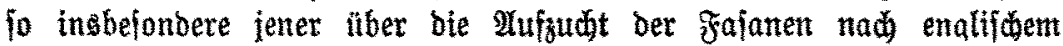

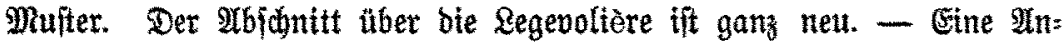
zahl von Beanftanbungen gelegentlid ber Bepreduntg ber 3. Yuffage ${ }^{1}$ ) baben Beatutung gefunden, uno barf bie neue Aluflage jebenfalls als eine verbefferte bezeidnet werden.

r.

Ir. 90.

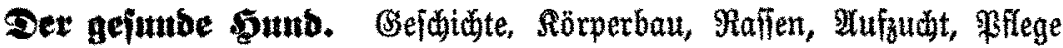
uttw. Des Sundes. Für Şundebefiber bearbeitet von Dr. Georg Müller, fgl fächj. Obermebizinalrat, o. Brofeffor uno Direttor ber Rlinif für tleinere Saustiere an ber fgl. Tierärźtliden Fुodfdule zu Dresben. 2., neu bearbeitete 2ruflage. Mit 78 Textabbilbungen. Bertin, Berlag

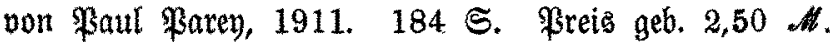

Die 1. Auflage biejes ber "Thaer-Bibliothef" zugehörigen $\mathfrak{B}$ ertes erfidien im \$abre 1899. Der Berf. hat bas Bub einer grünbliben Duraficht untergogen, neue abbilbungen aufgenommen, veraltete burch

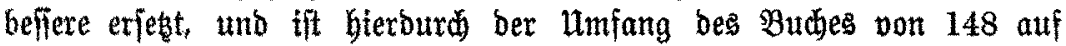
184 Setten, bie Babl ber Mbbilbungen won 64 auf 78 geftiegen. Der Berf. betont in Borwort noch bejonbers, baj er jelbitberfänolich bei feinen Atșfubrungen bemitht war, ben berechtigten Forderungen bes Tieridutes

1) Bergl. Forftw. Eentrtbl. 1896, \$. 357. 


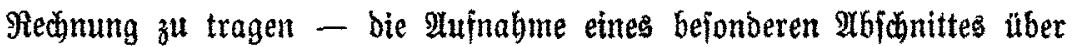
bie fohmerzloje Tötung bes Saunbes exiolgte aus biefem Grunbe.

Şundebefizern wirb bas gut auggeftattete Büdlein eine willfommene Gabe Fein.

ऽ.

Pr. 91.

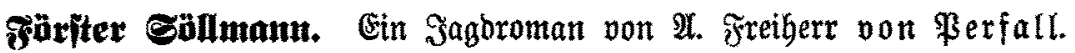

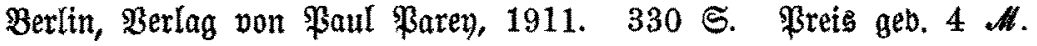

Der vorliegenbe, in bem 5yochgebirg an ber bayerifid:öfterreidjijhen

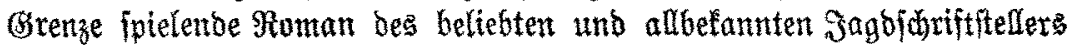

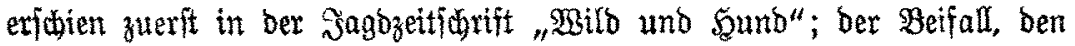

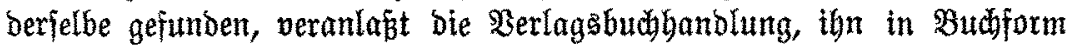
weiteren §reijen zugänglid zu mađen - ipeżiell in Gägertreifjen bürfte bie bramatidie Echtlderung ber unbeilvollen Folgen, welde bie sagd: leibenichaft in ben Scfichten ber bortigen Bevölferung nad) fich zieht, mit šntereffe gelejen werben.

$\mathfrak{N r} .92$.

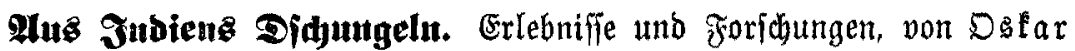
Raufnann. 2 Bände (360 Seiten) mit 2 Sarten, 12 Photogravüren und 265 Mbbildungen auf 152 Iafeln. Reipzig, Berlag von Rlinffarot \& Biermann, 1911. Freis eleg. geb. $20 \mu$.

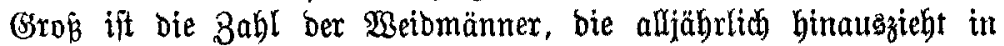
ferne Ränber, nad) Dem hohen Morden und bem heisen Süben, um bort zu jagen unb zu forichen; insbejonbere ift bas willoreide 2rfrifa bas ziel foldher Sagbreifen. (sin anberes Forfdingngegebiet bat fich ber Berfaffer des vorliegenden Berfes gewählt, bas hochinterefifante Sndien, uno was er bort auf 4 in ben Sahren 1901 - 1910 ausgetübrten Reifen gefehen uno erlebt bat, bae erzäblt ex in anregender $\mathfrak{B} 3$ eife, fid nidbt auf feine Jagberlebnifife uno ßejultate beidränteno, jondern aud eingebende Schilderungen von \&and und Reuten gebend.

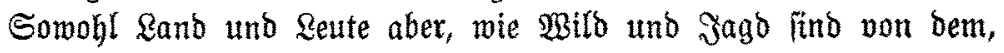
was uns burch afrifanifo jagoreifen betunnt geworben, auberorbentlid verichieben, unb curdjaus Reues tritt ung hiex entgegen. Dort ein Ranb von zum Teil boher filltur uno Entwidtung - man benfe an Stäbte wie Mabras, Bombey, Ralfutta - unb bier beibes erfit in ihren 2 In= fängen; bort bie bidilen, oft nabezu unburchoringliden Didungeln in Gegenjak zu ben ausgebehnten Steppen Iffrifas. Mußerorbentlid ver= fditeden ift denn aud bie Dierwelt, an welder, ingbejondere an Sropbilb,

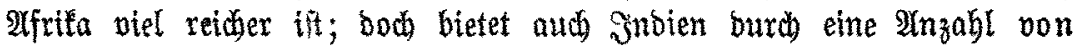


Tiergattungen bem Эäger großę Sntereffe, und mit joldhem folgen wir bem Berf. bei jeinen erfolgreichen ३agben auf Tiger uno \$anther, auf Elefanten unb ben mädtigen Baur, Den Bilbbiffel Yrfitens, auf Sambar= hiriche, Gazellen unb Milbldyeine. Die hierüber beigegebenen $\mathfrak{B i l d e r}$ müffen fich auf bas erlegte $\mathfrak{B i l b}$ bejobränten: bie bidte Didungel, ber geringere $\mathfrak{B i l b r e i c h t u m ~ m a c h e n ~} \mathfrak{A} u f n a h m e n$ lebenden $\mathfrak{B}$ Bilbes, wie fie vor allem Shillings in io vorirefflidjer $\mathfrak{B e i f e}$ gegeben, unmöglid.

Aber nidist mur bem $\mathfrak{B i l b}$, fonbern aud ben fo verjofiebenartigen

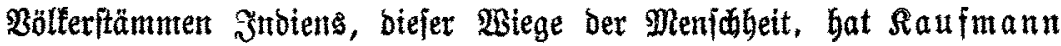
jeine $2 \mathfrak{A} u$ jmerffamfeit zugemendet unb fübrt fie uns in zablreidben guten

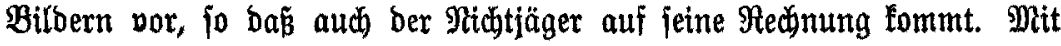
grober Inerfennung geoenlt ber Beri. audi bes gajffreien Entgegen= fommens unb ber tatfräftigen Antertiüzung, bie er jeitens ber Engländer, Privaten wie Bebörden, gefunben bat.

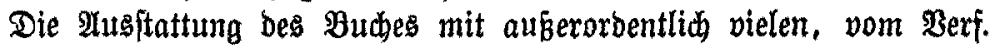
aufgenommenen und jehr gut wicbergegebenen 2 tbbildungen, wie burch Papier, Drud und Einband mús als eine vorzüglide bezeidnet werben.

\section{Hutizhen.}

Godihüulnałricht.

Der Pribatbozent ber Uniberfität Münden, exponterter Frorftnmtsaffeffor Dr.

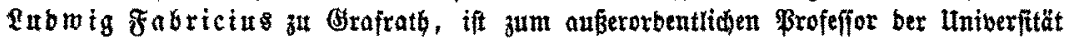

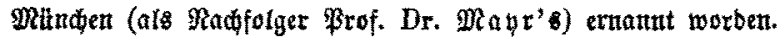

\section{Uuffallendes $\mathfrak{U}_{\mathfrak{u} f \text { treten }}$ der Sđ̆ütte.}

Sett bem $3 a h e 1891$ fïhre to bie 2 irtidaft in ben 130 ha umfaffenbent Warbungen ber अrbeitertolonie Simonahof in meiner Cigenidaft als Mitglieb ber Boritandichaft bes Bereins für Mrbeiterfolonieen in Bayern.

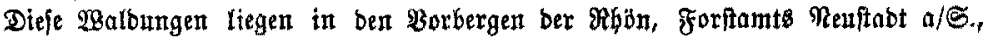

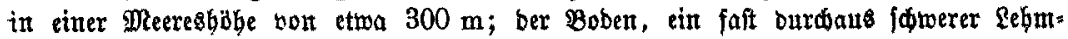
boben, gebërt ber oberften Sobidte bes Buntjanbfteins an unb war urfprïnglio vor= wiegenb mit Eiden beftodt, bie in Geftalt teils zienlid guten, inberwiegenb jebow

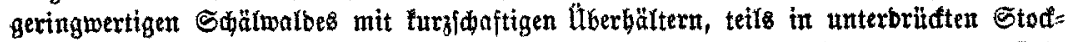
ausfdlägen in ben vorbanberen Fohrenbeftänben nod jeğt alfenthalben sorbanben fint.

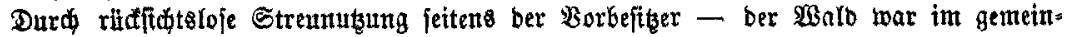
famen $\mathfrak{B e f t i s}$ von 4 Bautern, beren 4 Salfe ben Beiler Simonghof bitbeten - if ber Bobet faft butdans febr zuritfigetommen, verntagert unb verhetbet.

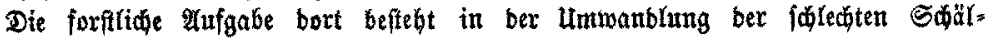

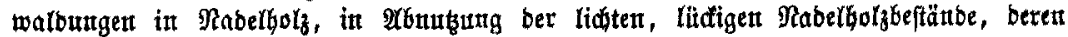
Mbtriebsertrag nur 70-80 fm auf ben Weftar beträgt, uno 2ufforftung ber Sitebs= 